\title{
Post-Partum Seizure from Subdural Hematoma Secondary to Cerebrospinal Fluid Leak Following Epidural Anesthesia
}

Anna Whalen-Browne, Kristyne Onizuka, Ragini Srinivasan, Maria Tiboni

\section{About the Authors}

Anna Whalen-Browne, Kristyne Onizuka, and Maria Tiboni are with the Department of Medicine, McMaster University, Hamilton, ON, Canada

Ragini Srinivasan, is with the Department of Neurology, McMaster University, Hamilton, ON, Canada

Author for correspondence: anna.whalen-browne@medportal.ca

Submitted: October 18, 2018. Accepted: February 8, 2019. Published: August 31, 2019.

\begin{abstract}
Headaches in the post-partum period are common, while new seizures are not. While many causes of post-partum headache are benign, it can be difficult to determine which patients warrant a more comprehensive workup to investigate for serious causes, which may only present later with neurologic sequelae such as new seizures. We suggest that persistent post-dural puncture headaches in the context of intrapartum epidural anesthesia can be suggestive of a serious cause such as intracranial hypotension or subdural hematoma. We describe a case of a 28 -year-old G1P1 female presenting with a first-time seizure at 19 days post-partum caused by subdural hematoma from a persistent cerebrospinal fluid leak. This was in the context of a history of typical post-dural puncture headache following delivery.
\end{abstract}

\section{RÉSUMÉ}

Les maux de tête dans la période post-partum sont fréquents, alors que les nouvelles crises ne le sont pas. Bien que de nombreuses causes de céphalées post-partum soient bénignes, il peut être difficile de déterminer quels patients justifient un bilan plus complet pour rechercher les causes graves, qui peuvent ne se présenter que plus tard avec des séquelles neurologiques comme de nouvelles crises. Nous suggérons que les maux de tête persistants post-ponctionnels dans le contexte de l’anesthésie épidurale intra-partum peuvent suggérer une cause grave comme l'hypotension intracrânienne ou un hématome sous-dural. Nous décrivons le cas d'une femme G1P1 de 28 ans qui présente une première crise d'épilepsie 19 jours après l'accouchement causée par un hématome sous-dural causé par une fuite persistante de liquide céphalorachidien. C'était dans le contexte d'une histoire de céphalées typiques après une ponction post-durale après l'accouchement.

Keywords: Post-partum, headache, post-dural puncture syndrome, seizure, subdural hematoma, pregnancy, epidural, anesthesia 
More than one-third of women suffer from headaches in the post-partum period, which can be due to a number of benign or concerning causes. ${ }^{1}$ As a period of physiologic flux and role adjustment, a high degree of clinical skill is required in order to discern which patients may benefit from more comprehensive workup. In contrast, new seizures in the post-partum period are uncommon and universally require serious investigation. We suggest that headache in the post-partum period, particularly with features of post-dural puncture headache (PDPH), can be a primary symptom of concerning intracranial pathology. If it is not identified early, such conditions may only be revealed when complications arise, such as new seizures. Therefore, we suggest that PDPH is an important clinical diagnosis and clue that can point us towards underlying pathology in the post-partum period.

Although generally a relatively safe practice, epidural anesthesia can be complicated by accidental dural puncture allowing leakage of cerebrospinal fluid (CSF) out of the subarachnoid space. Headache is the most common presentation of this procedural complication, which has been reported to be as common as $1-5 \%$ following spinal or epidural anesthesia, ${ }^{2}$ and as common as $75 \%$ of parturients in whom dural puncture was identified at the time of the procedure. ${ }^{3}$ The pathophysiology of PDPH is not fully understood but is thought to be related to loss of CSF volume causing intracranial hypotension, resulting in vessel vasodilatation and sagging of intracranial structures causing pain. ${ }^{4}$ In some cases, leakage of CSF from the site of dural puncture can be protracted, and lead to progressive intracranial hypotension, increasing the severity of PDPH, and further complications such as intracranial bleeding. Subarachnoid hemorrhage occurs at a rate of up to 6 per 100,000 deliveries, ${ }^{5}$ while subdural hematoma from tearing of dural bringing veins caused by hypotensive traction on the dura has more rarely been described. ${ }^{2}$ While a rare entity in the general post-partum population, this concerning complication can lead to devastating complications and must be kept in mind when assessing women in the post-partum period presenting with headache or seizure. We, therefore, suggest that a heightened degree of suspicion for women presenting with severe symptoms of PDPH in the context of known intrapartum epidural anesthesia may be warranted. Ultimately, we propose that this approach could prevent progression of dural puncture complication of epidural anesthesia to significant morbidity and mortality.

We describe a unique case of a 28 -year-old G1P1 female presenting with a first-time seizure at post-partum day 19 in the context of a PDPH with significant intracranial hypotension from protracted CSF leak. Imaging revealed a significant subdural hematoma collection as the likely epileptogenic focus, which was attributed to dural traction from intracranial hypotension following epidural anesthesia.

\section{Case Report}

A 28-year-old G1P1 female presented to the emergency department following a first-time generalized tonic-clonic seizure. She was 19-days post-partum from an induced vaginal delivery for postdates. She had no known medical history, including no prior seizures, and was on no medications. There were no concerning features for pre-eclampsia or eclampsia during pregnancy or following delivery, and there was no history of trauma.

This patient's labour and delivery had been largely uncomplicated, and she had received epidural anesthesia for pain relief during active labour. A few hours following delivery, the patient developed the onset of severe bilateral frontal headaches radiating to the occiput. She described positional symptoms including provocation with an upright position, and alleviation by lying supine. These headaches were quickly identified as likely being a post-dural puncture in etiology, and on post-partum day three, she was treated with a blood patch, which led to the resolution of these headaches. She then had a period of one week with no headaches until she began developing a new unique type of headache on post-partum day ten. At this time, she began noticing a progressive left-sided frontal headache of different quality, which she described as sharp and shooting, with a side locked quality. This headache remitted one week later, two days prior to her seizure at 19 days post-partum.

On presentation, the patient had no focal neurological symptoms and no injuries from her seizure. She was afebrile with a temperature of 36.6 degrees Celsius, normotensive with a blood pressure of $114 / 74$, with a heart rate of 86 beats per minute and respiratory rate of 12 breaths per minute, with oxygen saturation of $100 \%$ on room air. Apart from bite marks to the left lateral tongue, there were no abnormal findings on physical examination. Investigations including complete blood count, basic and extended electrolytes, liver enzymes, glucose, creatinine, urea, urine protein, electrocardiogram and chest radiography were all within the normal range. Computed tomography (CT) of the brain did, however, reveal a $1 \mathrm{~cm} \times 3.5 \mathrm{~cm}$ left superior posterior parietal subdural collection representing a subdural hematoma, along with mild mass effect on the subjacent brain parenchyma. The neurosurgery service was consulted but it was determined that the small size of the subdural hematoma would require no neurosurgical intervention unless there was radiologic evidence of enlarging volume SDH on repeat imaging. The neurology service was consulted regarding antiepileptic choice in the context of breastfeeding and to arrange ongoing outpatient follow up.

Based on her new onset seizures and structural focus, she was admitted to hospital for 48 hours for monitoring and initiation of antiepileptic medications. She was initially loaded with phenytoin in the emergency department, and then transitioned to levetiracetam for preferred side-effect profile and 
safety in breastfeeding. CT of her brain was repeated one-day post-admission and revealed no change in the size of a subdural hematoma or other epileptogenic focus.

She was discharged on levetiracetam and remained seizurefree. Magnetic resonance imaging with angiography of her brain three weeks post-discharge revealed subdural fluid collections consistent with subdural hematomas, unchanged when to prior CT. In keeping with intracranial hypotension, there was decreased mamillopontine distance which indicates downward displacement of the brain within the intracranial cavity. There were no other epileptogenic foci or vascular abnormalities noted.

\section{Discussion}

We present a case of post-partum headache and first-time seizure following a protracted CSF leak causing a subdural hematoma. This is in the context of epidural anesthesia at the time of labour and delivery.

Intracranial hypotension from protracted loss of CSF is a recognized phenomenon. Uncommonly, however, has this been shown to lead to dural traction and accumulation of hematomas in the subdural space due to damage to bridging vasculature. ${ }^{6,7}$ Once a subdural hematoma has accumulated, both headaches and new seizures are typical clinical features, as was the case in our patient of note. While there are a limited number of case reports of subdural hematoma secondary to significant intracranial hypotension following CSF leak post-dural puncture, there are no reported cases of this phenomenon causing new seizures in the post-partum period. ${ }^{8-10}$

Epidural anesthesia without complication involves the insertion of a catheter into the epidural space which lies between the ligamentum flavum and dura mater. This procedure can be complicated by puncture of the dura mater into the subarachnoid space, within which lie the spinal cord and CSF. If this occurs, CSF may leak from the subarachnoid space, causing a post-dural puncture syndrome characterized by typical symptoms including headache, nausea, photophobia, and more., ${ }^{2,11}$

Importantly, a number of known risk factors for PDPHs put our obstetric population at particularly high risk. These patient risk factors include female gender, ${ }^{12-14}$ pregnancy, ${ }^{15-17}$ active pushing in the second stage of labour, ${ }^{16,17}$ as well as the widespread use of epidural anesthesia in this population. Furthermore, unlike many procedural complications which become more frequent with increasing age, the age group at highest risk of PDPH is 20-30 year-olds, who have been shown to be at three to five times the risk as compared to adults over 60 years. ${ }^{2,18,19}$ Some data indicate that the incidence of PDPH in the obstetric population may be as high as $76-88 \%,{ }^{11,20,21}$ This is compared to the rate of unintentional dural puncture complicating epidural anesthesia in all subjects which has been reported with varying incidence of $0.19-4.4 \% .^{2}$ However, there is a lack of data describing the relationship between PDPH and subdural hematoma caused by dural traction from significant CSF loss. There also exist no clear universal guidelines to direct the investigation of women presenting with new post-partum headaches. ${ }^{22}$ While more often than not, these headaches will be of benign etiology, new headaches in the post-partum period, particularly in the context of epidural anesthesia at the time of labour, may, in fact, be the first clinical warning sign of significant sequelae to come.

The differential diagnosis of headaches following delivery includes common, less concerning, etiologies such as non-specific headache, caffeine-withdrawal headache, tension headache, sinus headache, and migraine. Headache in the postpartum period may also, however, be attributable to serious causes such as PDPH, preeclampsia, meningitis, intracranial hemorrhage, or cerebrovenous thrombosis. ${ }^{6,11}$ The typical PDPH is severe, throbbing in quality, frontal in location with radiation to the occiput, and characteristically positional with provocation while upright or sitting, and relief in the supine position. Criteria for low-pressure headaches, however, include headaches of any quality occurring in temporal relation with the development of low CSF pressure as seen on imaging or my imaging. Other clinical symptoms may include photophobia, nausea, vomiting, neck stiffness, tinnitus, and dizziness. ${ }^{2,5,11}$

This is important to take into account when considering our current practices in the investigation of women presenting with new headaches in the post-partum period. Acknowledgement and understanding of this phenomenon, as well as the potential consequences, might allow us to shape guidelines to incorporate the appropriate prompt investigation of women with high-risk features of PDPH in the post-partum period. A first obvious point which could help rule this out would be if the post-partum patient presenting with headache did not receive epidural anesthesia. While common post-partum history questions include the gestational date, type of delivery, the spontaneity of delivery, and complications at the time of delivery, many physicians never consider the type of anesthesia administered during labour and delivery. Other helpful questions for women presenting with headaches in the post-partum period revolve around the typical characteristics of the PDPH. In the patient presented in this report, she also presented with a second discrete type of headache following blood patching, which assumingly slowed her CSF leak and resolved her typical-PDPH. This second headache was characteristic of that caused by dural traction with a side locked quality. ${ }^{8,9}$ In our patient, this was described as the sensation of stretching of a nerve and was specific in location to the area overlying the radiologically-confirmed subdural hematoma.

We, therefore, believe that subdural hematoma from CSF leak should be on the differential diagnosis of postpartum headache and seizure, in any patient who has undergone epidural or spinal procedures that could have led to accidental dural puncture. 
These patients would then have to be risk-stratified based on clinical suspicion to determine who may warrant investigation for severe intracranial hypotension and associated complications. Benefits would include early detection of worrisome conditions such as severe intracranial hypotension or bleed, which have the potential to lead to devastating outcomes such as significant hemorrhage, seizure, mass effect, herniation, and death. While the more aggressive investigation of women presenting with PDPH following epidural anesthesia could allow for the earlier identification and treatment of women suffering from these consequences, there are also downsides to a more aggressive approach to investigation. This includes more women being exposed to radiation, specifically in the post-partum, as well as the risk of false positives, unnecessary worry, and initiation of treatment (antiepileptic therapy, neurosurgical intervention) in patients who may never have developed clinically significant sequelae.

This case highlights a rare but important presentation of seizure secondary to subdural hematoma caused by intracranial hypotension. This is in the context of a protracted CSF leak following a common complication of intrapartum epidural anesthesia. While few similar cases have been reported to date, subdural hematoma from the depletion of CSF is an essential diagnosis to have on the differential for all patients presenting with new headaches or seizures following spinal or epidural procedures. This is particularly pertinent in obstetric and postpartum populations, who commonly have a number of risk factors putting them at particular risk and routinely receive these procedures. While this case report highlights a potentially serious complication of significant CSF leakage following a dural puncture, further data on the incidence, diagnosis, and management of this complication would be valuable to ultimately help guide our care of this population moving forward.

\section{Contributorship}

This case report was written by AWB. All authors were directly involved in the patient's care and contributed to the editing of this report. All authors guarantee the accuracy and authenticity of this case.

\section{Ethical Approval}

No identifiable information was used in the publication of this article.

\section{Funding}

The author(s) received no financial support for the research, authorship, and/or publication of this article.

\section{Declaration of Interests}

The author(s) declare no potential conflicts of interest with respect to the research, authorship, and/or publication of this article.

\section{Acknowledgements}

The author(s) wish to acknowledge the members of the wider inter-disciplinary and inter-specialty healthcare team involved in the management of this case.

\section{References}

1. Goldszmidt E, Kern R, Chaput A, and Macarthur A. The incidence and etiology of postpartum headaches: a prospective cohort study. Obst and Ped Anesth 2005;52(9):971-77.

2. Bezov D, Lipton R and Ashina A. PDPH: Part I diagnosis, epidemiology, etiology, and pathophysiology. Headache 2010;50:1144-52.

3. Van de Velde M, Schepers R, Berends N, et al. Ten years of experience with accidental dural puncture and post-dural puncture headache in a tertiary obstetric anesthesia department. Int J Obstet Anesth 2009;17:329-35.

4. Algin O, Taskapilioglu O, Zan E, et al. Detection of CSF leaks with magnetic resonance imaging in intracranial hypotension syndrome. J Neurorad 2011;38:175-77.

5. Sachs A and Smiley R. Post-dural puncture headache: The worst common complication in obstetric anesthesia. Sem Perinat 2014;38:386-94.

6. Kueper M, Goericke S, and Kastup O. Cerebral venous thrombosis after epidural blood patch: Coincidence or causal relation? A case report and review of the literature. Cephalalgia 2008;28:769-73.

7. Monsalve G, Ortega R, and Rueda-Acevedo M. Multiple dural sinus thrombosis: A differential diagnosis of a pospuncture headache in a puerperal patient. Neurol India 2006;54:447-48.

8. Ramirez S, Gredilla E, Martinez B, and Gilsanz F. Bilateral subdural hematoma secondary to accidental dural puncture. Braz J Anesthesiol 2015;65(4):306-309.

9. Zeidan A, Farhat O, Maaliki H, et al. Does postdural puncture headache left untreated lead to subdural hematoma? Case report and review of the literature. Int J Obs Anesth 2006;15:50-58.

10. Liang $M$, and Pagel P. Bilateral interhemispheric subdural hematoma after inadvertent lumbar puncture in a parturient. Can J Anesth 2012;59:389-93.

11. Kuczkowski K. Post-dural puncture headache in the obstetric patient: An old problem. New solutions. Minerva Anesth 2004;70:823-30.

12. Khlebtovsky A, Weitzen S, Steiner I, et al. Risk factors for post lumbar puncture headache. Clin Neurol and Neurosurg 2015;131:78-81.

13. Wu C, Rowlingson A, Cohen S, et al. Gender and post-dural puncture headache. Anesthesiology 2006;105(3):613-18.

14. Amorim A, Gomes de Barros M and Valença M. Post-dural (postlumbar) puncture headache: Risk factors and clinical features. Cephalalgia 2012;32(12):916-23.

15. Stride P and Cooper G. Dural taps revisited. A 20-year survey from Birmingham Maternity Hospital. Anesthesia 1993;48(247).

16. Angle P, Thompson D, Halpern S and Wilson D. Second stage pushing correlates with headache after unintentional dural puncture in parturients. Can J Anaesth 1999;46(9):861-66.

17. Franz A, Jia S, Bahnson $H$ et al. The effect of second-stage pushing and body mass index of postdural puncture headache. J of Clin Anesth 2017;37:77-81.

18. Wadud R, Laiq N, Qureshi F, and Jan A. The frequency of postdural puncture headache in different age groups. J Coll Phys Surg Pak 2006;16(6):389-92.

19. Vilming S, Schrader H, and Monstad I. The significance of age, sex, and cerebrospinal fluid pressure in post-lumbar puncture headache. Cephalalgia 1989;9:99-106.

20. Banks S, Paech M, and Gurrin L. An audit of epidural blood path after accidental dural puncture with a Tuohy needle in obstetric patients. Int J Obstet Anesth 2001;10(3):172-76.

21. Sprigge J, and Harper S. Accidental dural puncture and post-dural puncture headache in obstetric anesthesia: presentation and management: a 23-year survey in a district general hospital. Anaesthesia 2008;63(1):36.

22. Stella C, Jodicke C, How H, et al. Postpartum headache: Is your work-up complete? Am J Obstet Gynecol 2007;196L 318e1-318e7. 